\title{
Extra Derivative Multistep Methods with Trigonometric-Fitting for Oscillatory Problems
}

\author{
${ }^{1}$ Sufia Zulfa Ahmad, ${ }^{1,3}$ Mohamed Yousif Turki, ${ }^{1,2}$ Fudziah Ismail and ${ }^{1,2}$ Norazak Senu \\ ${ }^{1}$ Department of Mathematics, Faculty of Science, \\ ${ }^{2}$ Institute for Mathematical Research, Universiti Putra Malaysia, 43400 Serdang, Selangor, Malaysia \\ ${ }^{3}$ Department of Mathematics, Faculty of Education and Pure Sciences, \\ Al-Anbar University, Ramadi, Iraq
}

\begin{abstract}
A set of new linear multistep method of order three and four with extra derivatives are developed for solving special second order ordinary differential equations. The extra derivatives are incorporated into the methods, so that, a more accurate numerical results can be obtained. The methods are developed using the sequence of Chebyshev polynomials as the basis function. The methods are then trigonometrically-fitted, so that, they are suitable for solving highly oscillatory problems arise from the special second order ordinary differential equations. Numerical experiments are carried out to show the efficiency and accuracy of the new methods in comparison with methods in the literature.
\end{abstract}

Key words: Linear multistep method, collocation method, trigonometrically-fitted, oscillatory problems, accuracy, different equation

\section{INTRODUCTION}

The special second order Ordinary Differential Equations (ODEs) can be represented by:

$$
y^{\prime \prime}=f(x, y), y\left(x_{0}\right)=x_{0}, y^{\prime}\left(x_{0}\right)=y_{0}^{\prime}
$$

in which the first derivative does not appear explicitly. This type of problems often appears in the field of science, mathematics and engineering such as quantum mechanics, spatial semi-discretizations of wave equations, populations modeling and celestial mechanics. The solutions of such differential equations often exhibit oscillatory properties and are harder to solve.

Equation 1 can be directly solved using Runge-Kutta Nystrom (RKN) methods which can be seen by Dormand etal. (1987) and Sommeijer(1987). Franco (1995) and Coleman (2003) also developed hybrid algorithm and constructed the order condition of hybrid method, respectively as a different approaches to directly solve Eq. 1. Recently, researchers practically used fitted methods such as phase-fitted and trigonometrically-fitted in order to enhance the efficiency of the original methods, so that, accurate numerical results can be obtained when the solutions to the problems are highly oscillatory. Some RKN and hybrid methods with various modification techniques for the integration of oscillatory problems can be seen by Papadopoulos et al. (2009), Kosti et al. (2012), Samat et al. (2011) and Ahmad et al. (2013a, b). On the other hand, the simplicity of the interpolation and collocation methods has caught the attention of various researchers to develop different types of collocation methods for solving (Eq. 1) such as the research of researchers by Guo (2007), Jator (2008) and Yap et al. (2014).

Hence, in this study, we developed a new extra derivative Linear Multistep Method (LMM) with collocation technique using Chebyshev polynomial as basis function. In order to improve the efficiency of the methods, we trigonometrically fitted the methods, so that, the coefficients will depend on the fitted frequency and step size of the problems.

\section{MATERIALS AND METHODS}

Derivation of LMM using collocation technique: The general k-step LMM for solving special second order ODEs is given as:

$$
\sum_{j=0}^{k} \alpha_{j} y_{n+j}=h^{2} \sum_{j=0}^{k} \beta_{j} f_{n+j}
$$

where, $f_{n+j}=y{ }_{n+j}, \alpha_{j}$ and $\beta_{j}$ are uniquely determined and $\alpha_{0}+\beta_{0} \neq 0, \alpha_{\mathrm{k}}=1$. Aboiyar et al. has constructed a LMM with collocation technique using Probabilist's Hermite polynomial as basis function for solving first order ODEs. Here, we will use Chebyshev polynomials as basis function. The following are the first five terms of the sequence from Chebyshev polynomials:

Corresponding Author: Sufia Zulfa Ahmad, Department of Mathematics, Faculty of Science, Universiti Putra Malaysia, 


$$
\left.\begin{array}{l}
\mathrm{T}_{0}(\mathrm{x})=1, \mathrm{~T}_{1}(\mathrm{x})=\mathrm{x}, \mathrm{T}_{2}(\mathrm{x})=2 \mathrm{x}^{2}-1 \\
\mathrm{~T}_{3}(\mathrm{x})=4 \mathrm{x}^{3}-3 \mathrm{x}, \mathrm{T}_{4}(\mathrm{x})=8 \mathrm{x}^{4}-8 \mathrm{x}^{2}+1
\end{array}\right\}
$$

In this study, we are going to develop linear multistep method with extra derivatives of the form of:

$$
\sum_{j=0}^{k} \alpha_{j} y_{n+j}=h^{2} \sum_{j=0}^{k} \mu_{j} f_{n+j}+h^{3} \sum_{j=0}^{k} \eta_{j} g_{n+j}
$$

where, $\alpha_{j}, \mu_{j}$ and $\eta_{j}$ are constant values, $f_{n+j}=y_{{ }_{n+j}}$ and $\mathrm{g}_{n+j}=\mathrm{y}{ }_{\mathrm{n}+\mathrm{j}}$. We proceed to approximate the exact solution $y(x)$ by the interpolating function of the form:

$$
y(x)=\sum_{j=0}^{n} \alpha_{j} T_{(n)}\left(x-x_{k}\right)
$$

which is the polynomial of degree $\mathrm{n}$ and satisfied Eq. 5:

$$
y^{\prime \prime}(x)=f\left(x, y(x), x_{k} \leq x \leq x_{k+p}\right), y\left(x_{k}\right)=y_{k}
$$

Derivation of LMMC (3): For $n=4$, Eq. 4 can be written as:

$$
\begin{aligned}
y(x)= & a_{0}+a_{1}\left(x-x_{k}\right)+a_{2}\left[\left(x-x_{k}\right)^{2}-1\right]+a_{3}\left[\left(x-x_{k}\right)^{3}-3\left(x-x_{k}\right)\right]+ \\
& a_{4}\left[\left(x-x_{k}\right)^{4}-8\left(x-x_{k}\right)^{2}+1\right]
\end{aligned}
$$

Differentiating Eq. 6, three times and we get the first, second and third derivatives of Eq. 7-9 as follows:

$$
\begin{aligned}
& y^{\prime}(x)= a_{1}+a_{2} 2\left(x-x_{k}\right)+a_{3} 3\left[\left(x-x_{k}\right)^{2}-1\right]+ \\
& a_{4} 4\left[\left(x-x_{k}\right)^{3}-4\left(x-x_{k}\right)\right] \\
& y^{\prime \prime}(x)= 2 a_{2}+a_{3} 6\left(x-x_{k}\right)+a_{4} 4\left[3\left(x-x_{k}\right)^{2}-4\right] \\
& y^{\prime \prime}(x)=6 a_{3}+a_{4} 24\left(x-x_{k}\right)
\end{aligned}
$$

Next, Eq. 6 and 8 are collocated at $x=x_{k+1}, x_{k+2}$ and interpolated Eq. 9 at $\mathrm{x}=\mathrm{x}_{\mathrm{k}+1}$ which yields:

$$
\begin{gathered}
y\left(x_{k+1}\right)=a_{0}+a_{1}\left(x_{k+1}-x_{k}\right)+a_{2}\left[\left(x_{k+1}-x_{k}\right)^{2}-1\right]+ \\
a_{3}\left[\begin{array}{l}
\left(x_{k+1}-x_{k}\right)^{3}- \\
3\left(x_{k+1}-x_{k}\right)
\end{array}\right]+a_{4}\left[\begin{array}{l}
\left(x_{k+1}-x_{k}\right)^{4}- \\
8\left(x_{k+1}-x_{k}\right)^{2}+1
\end{array}\right]=y_{k+1} \\
y\left(x_{k+2}\right)=a_{0}+a_{1}\left(x_{k+2}-x_{k}\right)+a_{2}\left[\left(x_{k+2}-x_{k}\right)^{2}-1\right]+ \\
a_{3}\left[\begin{array}{l}
\left(x_{k+2}-x_{k}\right)^{3}- \\
3\left(x_{k+2}-x_{k}\right)
\end{array}\right]+a_{4}\left[\begin{array}{l}
\left(x_{k+2}-x_{k}\right)^{4}- \\
8\left(x_{k+2}-x_{k}\right)^{2}+1
\end{array}\right]=y_{k+2} \\
y^{\prime \prime}\left(x_{k+1}\right)=2 a_{2}+a_{3} 6\left(x_{k+1}-x_{k}\right)+ \\
a_{4} 4\left[3\left(x_{k+1}-x_{k}\right)^{2}-4\right]=f_{k+1} \\
y^{\prime \prime}\left(x_{k+2}\right)=2 a_{2}+a_{3} 6\left(x_{k+2}-x_{k}\right)+ \\
a_{4} 4\left[3\left(x_{k+2}-x_{k}\right)^{2}-4\right]=f_{k+2} \\
y^{\prime \prime}\left(x_{k+1}\right)=6 a_{3}+a_{4} 24\left(x_{k+1}-x_{k}\right)=g_{k+1}
\end{gathered}
$$

By substituting $h=x_{k+1}-x_{k}$ and $2 h=x_{k+2}-x_{k}$ into Eq. 10-14, we obtain the following:

$$
\begin{gathered}
\mathrm{y}_{\mathrm{k}+1}=\mathrm{a}_{0}+(\mathrm{h}) \mathrm{a}_{1}+\mathrm{a}_{2}\left[\mathrm{~h}^{2}-1\right]+\mathrm{a}_{3}\left[\mathrm{~h}^{3}-3 \mathrm{~h}\right]+\mathrm{a}_{4}\left[\mathrm{~h}^{4}-8 \mathrm{~h}^{2}+1\right] \\
\mathrm{y}_{\mathrm{k}+2}=\mathrm{a}_{0}+(2 \mathrm{~h}) \mathrm{a}_{1}+\mathrm{a}_{2}\left[4 \mathrm{~h}^{2}-1\right]+\mathrm{a}_{3}\left[8 \mathrm{~h}^{3}-6 \mathrm{~h}\right]+ \\
\mathrm{a}_{4}\left[16 \mathrm{~h}^{4}-32 \mathrm{~h}^{2}+1\right] \\
\mathrm{f}_{\mathrm{k}+1}=2 \mathrm{a}_{2}+\mathrm{a}_{3}(6 \mathrm{~h})+\mathrm{a}_{4}\left[12 \mathrm{~h}^{2}-16\right] \\
\mathrm{f}_{\mathrm{k}+2}=2 \mathrm{a}_{2}+\mathrm{a}_{3}(12 \mathrm{~h})+\mathrm{a}_{4}\left[48 \mathrm{~h}^{2}-16\right] \\
\mathrm{g}_{\mathrm{k}+1}=6 \mathrm{a}_{3}+\mathrm{a}_{4}(24 \mathrm{~h})
\end{gathered}
$$

Rearranging Eq. 15-19 into matrix form as follows:

which can be simplified as:

$$
\left[\begin{array}{ccccc}
1 & h & h^{2}-1 & h^{3}-3 h & h^{4}-8 h^{2}+1 \\
1 & 2 h & 4 h^{2}-1 & 8 h^{3}-6 h & 16 h^{4}-16 h^{2}+1 \\
0 & 0 & 2 h & 6 h^{2} & 12 h^{2}-16 \\
0 & 0 & 2 h & 12 h^{2} & 48 h^{2}-16 \\
0 & 0 & 0 & 6 h & 24 h^{2}
\end{array}\right]\left[\begin{array}{c}
a_{0} \\
a_{1} \\
a_{2} \\
a_{3} \\
a_{4}
\end{array}\right]=\left[\begin{array}{c}
y_{k+1} \\
y_{k+2} \\
h_{k+1} \\
h f_{k+2} \\
h_{k+1}
\end{array}\right]
$$

$$
\mathrm{XA}=\mathrm{Y}
$$


Where:

$$
X=\left[\begin{array}{ccccc}
1 & h & h^{2}-1 & h^{3}-3 h & h^{4}-8 h^{2}+1 \\
1 & 2 h & 4 h^{2}-1 & 8 h^{3}-6 h & 16 h^{4}-16 h^{2}+1 \\
0 & 0 & 2 h & 6 h^{2} & 12 h^{2}-16 \\
0 & 0 & 2 h & 12 h^{2} & 48 h^{2}-16 \\
0 & 0 & 0 & 6 h & 24 h^{2}
\end{array}\right]
$$

From Eq. 20, we can solve for A, where:

$$
\mathrm{A}=\mathrm{X}^{-1} \mathrm{Y}
$$

Solving Eq. 21, the coefficients of $\mathrm{a}_{0}, \mathrm{a}_{1}, \mathrm{a}_{2}, \mathrm{a}_{3}$ and $\mathrm{a}_{4}$ are obtained in terms of $\mathrm{y}_{\mathrm{k}+1}, \mathrm{y}_{\mathrm{k}+2}, \mathrm{f}_{\mathrm{k}+1}, \mathrm{f}_{\mathrm{k}+2}$ and $\mathrm{g}_{\mathrm{k}+1}$ :

$$
\begin{aligned}
& \mathrm{a}_{0}=2 \mathrm{y}_{\mathrm{k}+1}-\mathrm{y}_{\mathrm{k}+2}+\frac{1}{12} \frac{10 \mathrm{~h}^{4}-7}{\mathrm{~h}^{2}} \mathrm{f}_{\mathrm{k}+1}+\frac{1}{12} \frac{2 \mathrm{~h}^{4}+6 \mathrm{~h}^{2}+7}{\mathrm{~h}^{2}} \mathrm{f}_{\mathrm{k}+2}-\frac{1}{12} \frac{2 \mathrm{~h}^{4}+12 \mathrm{~h}^{2}+7}{\mathrm{~h}^{2}} \mathrm{~g}_{\mathrm{k}+1} \\
& \mathrm{a}_{1}=-\frac{\mathrm{y}_{\mathrm{k}+1}}{\mathrm{~h}}+\frac{\mathrm{y}_{\mathrm{k}+2}}{\mathrm{~h}}-\frac{1}{12} \frac{13 \mathrm{~h}^{2}-12}{\mathrm{~h}} \mathrm{f}_{\mathrm{k}+1}-\frac{1}{12} \frac{5 \mathrm{~h}^{2}+12}{\mathrm{~h}} \mathrm{f}_{\mathrm{k}+2}+\frac{3}{4}\left(\mathrm{~h}^{2}+2\right) \mathrm{g}_{\mathrm{k}+1} \\
& \mathrm{a}_{2}=-\frac{2}{3} \frac{\mathrm{f}_{\mathrm{k}+1}}{\mathrm{~h}^{2}}+\frac{1}{6} \frac{3 \mathrm{~h}^{2}+4}{\mathrm{~h}^{2}} \mathrm{f}_{\mathrm{k}+2}-\frac{1}{3} \frac{\left(3 \mathrm{~h}^{2}+2\right)}{\mathrm{h}} \mathrm{g}_{\mathrm{k}+1} \\
& \mathrm{a}_{3}=\frac{1}{3} \frac{\mathrm{f}_{\mathrm{k}+1}}{\mathrm{~h}}-\frac{1}{3} \frac{\mathrm{f}_{\mathrm{k}+2}}{\mathrm{~h}}+\frac{1}{2} \mathrm{~g}_{\mathrm{k}+1} \\
& \mathrm{a}_{4}=-\frac{1}{12} \frac{\mathrm{f}_{\mathrm{k}+1}}{\mathrm{~h}^{2}}+\frac{1}{12} \frac{\mathrm{f}_{\mathrm{k}+2}}{\mathrm{~h}^{2}}-\frac{1}{12} \frac{\mathrm{g}_{\mathrm{k}+1}}{\mathrm{~h}}
\end{aligned}
$$

Substituting the coefficients into Eq. 6 and letting $\mathrm{x}=\mathrm{x}_{\mathrm{k}+3}$, we obtain the following equation:

$$
\begin{aligned}
& y\left(x_{k+3}\right)=\left(2 y_{k+1}-y_{k+2}+\frac{1}{12} \frac{10 h^{4}-7}{h^{2}} f_{k+1}+\frac{1}{12} \frac{2 h^{4}+6 h^{2}+7}{h^{2}} f_{k+2}-\frac{1}{12} \frac{2 h^{4}+12 h^{2}+7}{h^{2}} g_{k+1}\right)+ \\
&\left(-\frac{y_{k+1}}{h}+\frac{y_{k+2}}{h}-\frac{1}{12} \frac{13 h^{2}-12}{h} f_{k+1}-\frac{1}{12} \frac{5 h^{2}+12}{h} f_{k+2}+\frac{3}{4}\left(h^{2}+2\right) g_{k+1}\right)\left(x_{k+3}-x_{k}\right)+ \\
&\left(-\frac{2}{3} \frac{f_{k+1}}{h^{2}}+\frac{1}{6} \frac{3 h^{2}+4}{h^{2}} f_{k+2}-\frac{1}{3} \frac{\left(3 h^{2}+2\right)}{h} g_{k+1}\right)\left[\left(x_{k+3}-x_{k}\right)^{2}-1\right]+ \\
&\left(\frac{1}{3} \frac{f_{k+1}}{h}-\frac{1}{3} \frac{f_{k+2}}{h}+\frac{1}{2} g_{k+1}\right)\left[\left(x_{k+3}-x_{k}\right)^{3}-3\left(x_{k+3}-x_{k}\right)\right]+\left(\frac{1}{3} \frac{f_{k+1}}{h}-\frac{1}{3} \frac{f_{k+2}}{h}+\frac{1}{2} g_{k+1}\right)\left[\left(x_{k+3}-x_{k}\right)^{4}-\right. \\
&\left.8\left(x_{k+3}-x_{k}\right)^{2}+1\right]=y_{k+3}
\end{aligned}
$$

Letting $3 h=\left(\mathrm{x}_{\mathrm{k}+3}-\mathrm{x}_{\mathrm{k}}\right)$, we obtain the discrete form of LMMC as:

$$
y_{k+3}=2 y_{k+2}-y_{k+1}+h^{2}\left(-\frac{1}{6} f_{k+1}+7 f_{k+2}\right)+h^{3}\left(-\frac{1}{6} g_{k+1}\right)
$$

\section{Order and Consistency of LMMC method}

Definition 1; Lambert (1973): The linear difference operator L is defined by:

$$
L[y(x) ; h]=\sum_{j=0}^{k}\left[\alpha_{j} y(x+j h)-h^{2} \mu_{j} f(x+j h)-h^{3} \eta_{j} g(x+j h)\right]
$$

where, $\mathrm{y}(\mathrm{x})$ is an arbitrary function that is sufficiently differentiable on $[\mathrm{a}, \mathrm{b}]$. By expanding the test function and its first derivative as Taylor series about $\mathrm{x}$ and collecting the terms to obtain:

$$
\mathrm{L}[\mathrm{y}(\mathrm{x}) ; \mathrm{h}]=\mathrm{c}_{0} \mathrm{y}(\mathrm{x})+\mathrm{c}_{1} \mathrm{hy}^{(\mathrm{x})}+, \ldots,+\mathrm{c}_{\mathrm{q}} \mathrm{h}^{(\mathrm{q})} \mathrm{y}^{(\mathrm{q})}(\mathrm{x})+, \ldots
$$


where, the coefficients of $c_{q}$ are constants independent of $\mathrm{y}(\mathrm{x})$. In particular:

$$
\left.\begin{array}{l}
c_{0}=\sum_{j=0}^{k} \alpha_{j}, \quad c_{1}=\sum_{j=0}^{k}\left(j \alpha_{j}\right) \\
c_{2}=\sum_{j=0}^{k}\left(\frac{j^{(2)}}{2 !} \alpha_{j}-\mu_{j}\right) \\
c_{3}=\sum_{j=0}^{k}\left(\frac{j^{(3)}}{3 !} \alpha_{j}-j \mu_{j}-\eta_{j}\right) \\
c_{q}=\sum_{j=0}^{k}\left(\frac{j^{(q)}}{q !} \alpha_{j}-\frac{j^{(q-2)}}{(q-2) !} \mu_{j}-\frac{j^{(q-3)}}{(q-3) !} \eta_{j}\right)
\end{array}\right\}
$$

Definition 2; Order of the method (Henrici, 1962): The associated linear multistep method (Eq. 22) is said to be of the order $\rho$ if, $c_{0}=c_{1}=\ldots=c_{\rho+1}=0$ and $c_{\rho+2} \neq 0$.

Definition 3; Consistency of the method: The method is said to be consistence if it has order at least one. In order to find the order and consistency of LMMC, we compare equation in Definition 1 with Eq. 23. We obtain the coefficients of:

$$
\alpha_{0}=1, \alpha_{1}=-2, \alpha_{2}=1, \mu_{0}=-\frac{1}{6}, \mu_{1}=\frac{7}{6}, \eta_{0}=-\frac{1}{6}
$$

By substituting the coefficients into Eq. 23, we obtain:

$$
\mathrm{c}_{0}=\mathrm{c}_{1}=\mathrm{c}_{2}=\mathrm{c}_{3}=\mathrm{c}_{4}=0 \text { and } \mathrm{c}_{5}=\frac{1}{8}
$$

Hence, the new method has order $\mathrm{p}=3$, it is consistent, since, it has order $\mathrm{p}>1$, thus, it is convergent. The method is denoted as linear multistep method with extra derivative using collocation technique of order three (LMMC (3)).

\section{Derivation of LMMC (4) order and consistency of the} method: In this study, we derive the LMMC of order four. For $\mathrm{n}=5$, we obtain Eq. 4 as:

$$
\begin{aligned}
y(x)= & a_{0}+a_{1}\left(x-x_{k}\right)+a_{2}\left[\left(x-x_{k}\right)^{2}-1\right]+ \\
& a_{3}\left[\left(x-x_{k}\right)^{3}-3\left(x-x_{k}\right)\right]+a_{4}\left[\left(x-x_{k}\right)^{4}-8\left(x-x_{k}\right)^{2}+1\right]+ \\
& a_{5}\left[\left(x-x_{k}\right)^{5}-20\left(x-x_{k}\right)^{3}+5\left(x-x_{k}\right)\right]
\end{aligned}
$$

Differentiating Eq. 26, three times gives:

$$
\begin{aligned}
& y^{\prime}(x)=a_{1}+a_{2} 2\left(x-x_{k}\right)+a_{3} 3\left[\left(x-x_{k}\right)^{2}-1\right]+ \\
& a_{4} 4\left[\left(x-x_{k}\right)^{3}-4\left(x-x_{k}\right)\right]+a_{5} 5\left[\left(x-x_{k}\right)^{4}-12\left(x-x_{k}\right)^{2}+1\right] \\
& y^{\prime \prime}(x)=2 a_{2}+a_{3} 6\left(x-x_{k}\right)+a_{4} 4\left[3\left(x-x_{k}\right)^{2}-4\right]+(26) \\
& a_{5} 20\left[\left(x-x_{k}\right)^{3}-6\left(x-x_{k}\right)\right] \\
& y^{\prime \prime \prime}(x)=6 a_{3}+a_{4} 24\left(x-x_{k}\right)+a_{5} 60\left[\left(x-x_{k}\right)^{2}-2\right]
\end{aligned}
$$

Equation 26 and 28 are collocated at $\mathrm{x}=\mathrm{x}_{\mathrm{k}+1}, \mathrm{x}_{\mathrm{k}+2}$ and Eq. 27 at $\mathrm{x}=\mathrm{x}_{\mathrm{k}+1}, \mathrm{x}_{\mathrm{k}+3}$ which yields:

$$
\begin{aligned}
y_{k+1}= & a_{0}+(h) a_{1}+a_{2}\left[h^{2}-1\right]+a_{3}\left[h^{3}-3 h\right]+ \\
& a_{4}\left[h^{4}-8 h^{2}+1\right]+a_{5}\left[h^{5}-20 h^{2}+5 h\right]
\end{aligned}
$$

$$
\begin{gathered}
\mathrm{y}_{\mathrm{k}+2}=\mathrm{a}_{0}+(2 \mathrm{~h}) \mathrm{a}_{1}+\mathrm{a}_{2}\left[4 \mathrm{~h}^{2}-1\right]+\mathrm{a}_{3}\left[8 \mathrm{~h}^{3}-6 \mathrm{~h}\right]+ \\
\mathrm{a}_{4}\left[16 \mathrm{~h}^{4}-32 \mathrm{~h}^{2}+1\right]+\mathrm{a}_{5}\left[32 \mathrm{~h}^{5}-160 \mathrm{~h}^{3}+10 \mathrm{~h}\right] \\
\mathrm{f}_{\mathrm{k}+2}=2 \mathrm{a}_{2}+\mathrm{a}_{3}(6 \mathrm{~h})+\mathrm{a}_{4}\left[12 \mathrm{~h}^{2}-16\right]+\mathrm{a}_{5}\left[20 \mathrm{~h}^{3}-120 \mathrm{~h}\right] \\
\mathrm{f}_{\mathrm{k}+3}=2 \mathrm{a}_{2}+\mathrm{a}_{3}(18 \mathrm{~h})+\mathrm{a}_{4}\left[48 \mathrm{~h}^{2}-16\right]+\mathrm{a}_{5}\left[540 \mathrm{~h}^{3}-360 \mathrm{~h}\right] \\
\mathrm{g}_{\mathrm{k}+1}=6 \mathrm{a}_{3}+\mathrm{a}_{4}(24 \mathrm{~h})+\mathrm{a}_{5}\left(60 \mathrm{hh}^{2}-120\right) \\
\mathrm{g}_{\mathrm{k}+2}=6 \mathrm{a}_{3}+\mathrm{a}_{4}(48 \mathrm{~h})+\mathrm{a}_{5}\left(240 \mathrm{hh}^{2}-120\right)
\end{gathered}
$$

Equation 29-34 can be written in matrix form as follows:

$$
\mathrm{XA}=\mathrm{Y}
$$

Where:

$$
\mathrm{X}=\left[\begin{array}{cccccc}
1 & \mathrm{~h} & \mathrm{~h}^{2}-1 & \mathrm{~h}^{3}-3 \mathrm{~h} & \mathrm{~h}^{4}-8 \mathrm{~h}^{2}+1 & \mathrm{~h}^{5}-20 \mathrm{~h}^{3}+5 \mathrm{~h} \\
1 & 2 \mathrm{~h} & 4 \mathrm{~h}^{2}-1 & 8 \mathrm{~h}^{3}-6 \mathrm{~h} & 16 \mathrm{~h}^{4}-32 \mathrm{~h}^{2}+1 & 32 \mathrm{~h}^{5}-160 \mathrm{~h}^{3}+10 \mathrm{~h} \\
0 & 0 & 2 \mathrm{~h} & 12 \mathrm{~h}^{2} & 48 \mathrm{~h}^{3}-16 \mathrm{~h} & 160 \mathrm{~h}^{4}-240 \mathrm{~h}^{2} \\
0 & 0 & 2 \mathrm{~h} & 18 \mathrm{~h}^{2} & 108 \mathrm{~h}^{3}-16 \mathrm{~h} & 540 \mathrm{~h}^{4}-360 \mathrm{~h}^{2} \\
0 & 0 & 0 & 6 \mathrm{~h} & 24 \mathrm{~h}^{2} & 60 \mathrm{~h}^{3}-120 \mathrm{~h} \\
0 & 0 & 0 & 6 \mathrm{~h} & 48 \mathrm{~h}^{2} & 240 \mathrm{~h}^{3}-120 \mathrm{~h}
\end{array}\right]
$$




$$
\begin{aligned}
\mathrm{A} & =\left[\begin{array}{llllll}
\mathrm{a}_{0} & \mathrm{a}_{1} & \mathrm{a}_{2} & \mathrm{a}_{3} & \mathrm{a}_{4} & \mathrm{a}_{5}
\end{array}\right]^{\mathrm{T}} \\
\mathrm{Y} & =\left[\begin{array}{llllll}
\mathrm{y}_{\mathrm{k}+1} & \mathrm{y}_{\mathrm{k}+2} & \mathrm{hf}_{\mathrm{k}+2} & \mathrm{hf}_{\mathrm{k}+3} & \mathrm{hg}_{\mathrm{k}+1} & \mathrm{hg}_{\mathrm{k}+2}
\end{array}\right]^{\mathrm{T}}
\end{aligned}
$$

Solving Eq. 35, we obtained the coefficients of $a_{0}-a_{4}$ and $a_{5}$ in terms of $y_{k+1}, y_{k+2}, f_{k+2}, f_{k+3} g_{k+1}$ and $g_{k+2}$ :

$$
\begin{gathered}
\mathrm{a}_{0}=2 \mathrm{y}_{\mathrm{k}+1}-\mathrm{y}_{\mathrm{k}+2}+\frac{3\left(6 \mathrm{~h}^{4}+6 \mathrm{~h}^{2}+7\right)}{20 \mathrm{~h}^{2}} \mathrm{f}_{\mathrm{k}+2}+\frac{\left(2 \mathrm{~h}^{4}-8 \mathrm{~h}^{2}-21\right)}{20 \mathrm{~h}^{2}} \mathrm{f}_{\mathrm{k}+3}-\frac{\left(32 \mathrm{~h}^{4}+72 \mathrm{~h}^{2}+49\right)}{60 \mathrm{~h}} \mathrm{~g}_{\mathrm{k}+1}-\frac{\left(17 \mathrm{~h}^{4}-18 \mathrm{~h}^{2}-56\right)}{30 \mathrm{~h}} \mathrm{~g}_{\mathrm{k}+2} \\
\mathrm{a}_{1}=-\frac{1}{\mathrm{~h}} \mathrm{y}_{\mathrm{k}+1}+\frac{1}{\mathrm{~h}} \mathrm{y}_{\mathrm{k}+2}-\frac{\left(153 \mathrm{~h}^{4}+120 \mathrm{~h}^{2}+110\right)}{100 \mathrm{~h}^{3}} \mathrm{f}_{\mathrm{k}+2}+\frac{\left(3 \mathrm{~h}^{4}+120 \mathrm{~h}^{2}+100\right)}{100 \mathrm{~h}^{3}} \mathrm{f}_{\mathrm{k}+3}+\frac{\left(392 \mathrm{~h}^{4}+480 \mathrm{~h}^{2}+165\right)}{300 \mathrm{~h}^{2}} \mathrm{~g}_{\mathrm{k}+1}+\frac{\left(149 \mathrm{~h}^{4}-690 \mathrm{~h}^{2}-495\right)}{300 \mathrm{~h}^{2}} \mathrm{~g}_{\mathrm{k}+2} \\
\mathrm{a}_{2}=\frac{3\left(3 \mathrm{~h}^{2}+4\right)}{10 \mathrm{~h}^{2}} \mathrm{f}_{\mathrm{k}+2}-\frac{2\left(\mathrm{~h}^{2}+3\right)}{5 \mathrm{~h}^{2}} \mathrm{f}_{\mathrm{k}+3}-\frac{2\left(9 \mathrm{~h}^{2}+7\right)}{15 \mathrm{~h}^{2}} \mathrm{~g}_{\mathrm{k}+1}+\frac{\left(9 \mathrm{~h}^{2}+32\right)}{15 \mathrm{~h}^{2}} \mathrm{~g}_{\mathrm{k}+2} \\
\mathrm{a}_{3}=-\frac{2\left(\mathrm{~h}^{2}+1\right)}{5 \mathrm{~h}^{2}} \mathrm{f}_{\mathrm{k}+2}+\frac{2\left(\mathrm{~h}^{2}+1\right)}{5 \mathrm{~h}^{2}} \mathrm{f}_{\mathrm{k}+3}+\frac{\left(8 \mathrm{~h}^{2}+3\right)}{15 \mathrm{~h}^{2}} \mathrm{~g}_{\mathrm{k}+1}-\frac{\left(23 \mathrm{~h}^{2}+18\right)}{30 \mathrm{~h}^{2}} \mathrm{~g}_{\mathrm{k}+2} \\
\mathrm{a}_{4}=\frac{3}{20 \mathrm{~h}^{2}} \mathrm{f}_{\mathrm{k}+2}-\frac{3}{20 \mathrm{~h}^{2}} \mathrm{f}_{\mathrm{k}+3}-\frac{7}{60 \mathrm{~h}} \mathrm{~g}_{\mathrm{k}+1}+\frac{4}{15 \mathrm{~h}} \mathrm{~g}_{\mathrm{k}+2} \\
\mathrm{a}_{5}=-\frac{1}{50 \mathrm{~h}^{3}} \mathrm{f}_{\mathrm{k}+2}+\frac{1}{50 \mathrm{~h}^{3}} \mathrm{f}_{\mathrm{k}+3}+\frac{1}{100 \mathrm{~h}^{2}} \mathrm{~g}_{\mathrm{k}+1}-\frac{3}{100 \mathrm{~h}^{2}} \mathrm{~g}_{\mathrm{k}+2}
\end{gathered}
$$

We substitute $a_{0}, a_{1}-a_{4}$ and $a_{5}$ into Eq. 35 and by letting $\mathrm{x}=\mathrm{x}_{\mathrm{k}+3}$ and $3 \mathrm{~h}=\left(\mathrm{x}_{\mathrm{k}+3}-\mathrm{x}_{\mathrm{k}}\right)$, we obtain the discrete form of LMMC as:

$$
\mathrm{y}_{\mathrm{k}+3}=2 \mathrm{y}_{\mathrm{k}+2}-\mathrm{y}_{\mathrm{k}+1}+\frac{\mathrm{h}^{2}}{10}\left(9 \mathrm{f}_{\mathrm{k}+2}+\mathrm{f}_{\mathrm{k}+3}\right)-\frac{\mathrm{h}^{3}}{30}\left(\mathrm{~g}_{\mathrm{k}+1}+2 \mathrm{~g}_{\mathrm{k}+2}\right)
$$

In order to find the order and consistency of the LMMC, we compare equation in definition 1 with Eq. 36. We obtain the coefficients of:

$$
\alpha_{0}=1, \alpha_{1}=-2, \alpha_{2}=1, \mu_{1}=\frac{9}{10}, \mu_{2}=\frac{1}{10}, \eta_{0}=-\frac{1}{30}, \eta_{1}=-\frac{1}{15}
$$

By substituting back the coefficients into Eq. 23, we obtain:

$$
\mathrm{c}_{0}=\mathrm{c}_{1}=\mathrm{c}_{2}=\mathrm{c}_{3}=\mathrm{c}_{4}=\mathrm{c}_{5}=0 \text { and } \mathrm{c}_{6}=-\frac{1}{144}
$$

From definition 2, the new method is considered as having order $\mathrm{p}=4$. The new method is consistent, since, the order $\mathrm{p}>1$. The method is denoted as linear multistep method with extra derivative using collocation technique of order four (LMMC (4)).

Trigonometrically-fitting the methods: In this study, we adapt the trigonometrically-fitting technique to LMMC
(3). By letting some of the coefficients to be unknown values of $k_{i}$, for $I=1,2,3$, LMMC (3) in general form can be written as follows:

$$
y_{n+1}=2 y_{n}-y_{n-1}+h^{2}\left(k_{1} f_{n-1}+k_{2} f_{n}\right)+h^{3}\left(k_{3} g_{n-1}\right)
$$

Integrating Eq. 37 using the linear combination of the functions $\{\sin (v x), \cos (v x)\}$ for $v \in R$. We obtain the following Eq. 38:

$$
\left.\begin{array}{c}
\cos (\mathrm{H})=2-\cos (\mathrm{H})-\mathrm{H}^{2}\left(\mathrm{k}_{1} \cos (\mathrm{H})+\mathrm{k}_{2}+\mathrm{k}_{3} \mathrm{H} \sin (\mathrm{H})\right) \\
\mathrm{k}_{1} \sin (\mathrm{H})=\mathrm{k}_{3} \mathrm{H} \cos (\mathrm{H})
\end{array}\right\}
$$

Where:

$\mathrm{H}=v \mathrm{~h}$

$\mathrm{h}=$ The step size

$v=$ The fitted frequency

Solving Eq. 38 and letting, $k_{1}=-1 / 6$, the value of the remaining coefficients is obtain in terms of $\mathrm{H}$ :

$$
\begin{aligned}
\mathrm{k}_{2}= & \frac{7}{6}+\frac{3}{80} \mathrm{H}^{4}+\frac{851}{60480} \mathrm{H}^{6}+\frac{20777}{3628800} \mathrm{H}^{8}+ \\
& \frac{7939}{3421440} \mathrm{H}^{10}+\mathrm{O}\left(\mathrm{H}^{12}\right)
\end{aligned}
$$


$\mathrm{k}_{3}=-\frac{1}{6}-\frac{1}{18} \mathrm{H}^{2}-\frac{1}{45} \mathrm{H}^{4}-\frac{17}{1890} \mathrm{H}^{6}-\frac{31}{8505} \mathrm{H}^{8}-\frac{691}{467775} \mathrm{H}^{10}+\mathrm{O}\left(\mathrm{H}^{12}\right)$

This new method is denoted as trigonometricallyfitted linear multistep method with extra derivative using collocation technique of order three (TF-LMMC (3)).

Then we apply the trigonometrically-fitting technique to LMMC (4). By letting some of the coefficients to be unknown values of $k_{i}$, for $i=1,2,3,4$, rewrite the formula in general form, we have:

$$
\mathrm{y}_{\mathrm{n}+1}=2 \mathrm{y}_{\mathrm{n}}-\mathrm{y}_{\mathrm{n}-1}+\mathrm{h}^{2}\left(\mathrm{k}_{1} \mathrm{f}_{\mathrm{n}}+\mathrm{k}_{2} \mathrm{f}_{\mathrm{n}+1}\right)+\mathrm{h}^{3}\left(\mathrm{k}_{3} \mathrm{~g}_{\mathrm{n}-1}+\mathrm{k}_{4} \mathrm{~g}_{\mathrm{n}}\right)
$$

We integrate Eq. 39 using the linear combination of the function $\{\sin (v h), \cos (v h)\}$ for $v \in \mathrm{R}$. Therefore, the following equation are obtained:

$$
\left.\begin{array}{c}
\cos (\mathrm{H})=2-\cos (\mathrm{H})-\mathrm{H}^{2}\left(\mathrm{k}_{1}+\mathrm{k}_{2} \cos (\mathrm{H})+\mathrm{k}_{3} \mathrm{H} \sin (\mathrm{H})\right) \\
\mathrm{k}_{2} \sin (\mathrm{H})=-\mathrm{H}\left[\mathrm{k}_{3} \cos (\mathrm{H})+\mathrm{k}_{4}\right]
\end{array}\right\}
$$

Where:

$\mathrm{H}=v \mathrm{~h}$

$\mathrm{h}=$ The step size

$v=$ The fitted frequency

Solving Eq. 40 simultaneously by letting $\mathrm{k}_{1}=9 / 10$ and $\mathrm{k}_{3}=-1 / 30$ the value of the remaining coefficients is obtained in terms of $\mathrm{H}$ as follows:

$$
\begin{aligned}
\mathrm{k}_{2}= & \frac{1}{10}-\frac{1}{144} \mathrm{H}^{4}-\frac{313}{100800} \mathrm{H}^{6}-\frac{923}{725760} \mathrm{H}^{8}- \\
& \frac{6437}{12474000} \mathrm{H}^{10}+\mathrm{O}\left(\mathrm{H}^{12}\right) \\
\mathrm{k}_{4}=-\frac{1}{15}+ & \frac{3}{400} \mathrm{H}^{4}+\frac{83}{432000} \mathrm{H}^{6}+\frac{983}{1209600} \mathrm{H}^{8}+\mathrm{O}\left(\mathrm{H}^{10}\right)
\end{aligned}
$$

This new method is denoted as trigonometricallyfitted linear multistep method with extra derivative using collocation technique of order four (TF-LMMC(4)).

\section{RESULTS AND DISCUSSION}

In this study, the new methods $\operatorname{LMMC}(3,4)$, TF-LMMC $(3,4)$ are tested for problems. The 1-6 in order to show the methods capability for solving oscillatory problems. The methods are compared using a measure of the accuracy, Absolute error which is defined by:

$$
\text { Absolute error }=\max \left\{\left\|y\left(x_{n}\right)-y_{n}\right\|\right\}
$$

Where:

$$
\begin{aligned}
& y\left(x_{n}\right)=\text { The exact solution } \\
& y_{n}=\text { The computed solution }
\end{aligned}
$$

The test problems are listed as below.

Problem 1; An almost periodic orbit problem studied by Stiefel and Bettis (1969):

$$
\begin{gathered}
y_{1}^{\prime \prime}(x)+y_{1}(x)=0.001 \cos (x), y_{1}(0)=1, y_{1}^{\prime}(0)=0 \\
y_{2}^{\prime \prime}(x)+y_{2}(x)=0.001 \sin (x), y_{2}(0)=0, y_{2}^{\prime}(0)=0.9995
\end{gathered}
$$

Exact solution is $\mathrm{y}_{1}=\cos (\mathrm{x})+0.0005 \mathrm{x} \sin (\mathrm{x})$ and $\mathrm{y}_{2}=\sin (\mathrm{x})-0.0005 \mathrm{x} \cos (\mathrm{x})$. The fitted frequency is $\omega=1$.

Problem 2; In homogeneous system by Lambert and Watson (1976):

$$
\begin{gathered}
\frac{d^{2} y_{1}(x)}{d t^{2}}=-\omega^{2} y_{1}(x)+\omega^{2} f(x)+f^{\prime \prime}(x), y_{1}(0)=a+f(0) \\
y_{1}^{\prime}(0)=f^{\prime}(0) \\
\frac{d^{2} y_{2}(x)}{d t^{2}}=-\omega^{2} y_{2}(x)+\omega^{2} f(x)+f^{\prime \prime}(x), y_{2}(0)=f(0) \\
y_{2}^{\prime}(0)=\omega a+f^{\prime}(0)
\end{gathered}
$$

Where:

$$
\begin{array}{ll}
\mathrm{f}(\mathrm{x}) & =\mathrm{e}^{-0.05 \mathrm{x}} \\
\mathrm{a} & =0.1 \\
\mathrm{y}_{1}(\mathrm{x}) & =\operatorname{acos}(\omega \mathrm{x})+\mathrm{f}(\mathrm{x}) \\
\mathrm{y}_{2}(\mathrm{x}) & =\operatorname{asin}(\omega \mathrm{x})+\mathrm{f}(\mathrm{x})
\end{array}
$$

The fitted frequency is $\omega=20$.

Problem 3; In homogeneous system studied by Franco (2006):

$$
\begin{gathered}
y^{\prime \prime}(x)=\left(\begin{array}{cc}
\frac{101}{2} & -\frac{99}{2} \\
-\frac{99}{2} & \frac{101}{2}
\end{array}\right) y(x)=\delta\left(\begin{array}{cc}
\frac{93}{2} \cos (2 x) & -\frac{99}{2} \sin (2 x) \\
\frac{93}{2} \sin (2 x) & -\frac{99}{2} \cos (2 x)
\end{array}\right) \\
y(0)=\left(\begin{array}{c}
-1+\delta \\
1
\end{array}\right), y^{\prime}(0)=\left(\begin{array}{c}
-10 \\
10+2 \delta
\end{array}\right) \delta=10^{-3}
\end{gathered}
$$

Exact solution is given by:

$$
y(t)=\left(\begin{array}{c}
-\cos (10 x)-\sin (10 x)+\delta \cos (2 x) \\
\cos (10 x)+\sin (10 x)+\delta \sin (2 x)
\end{array}\right)
$$

The fitted frequency is $=10$. 


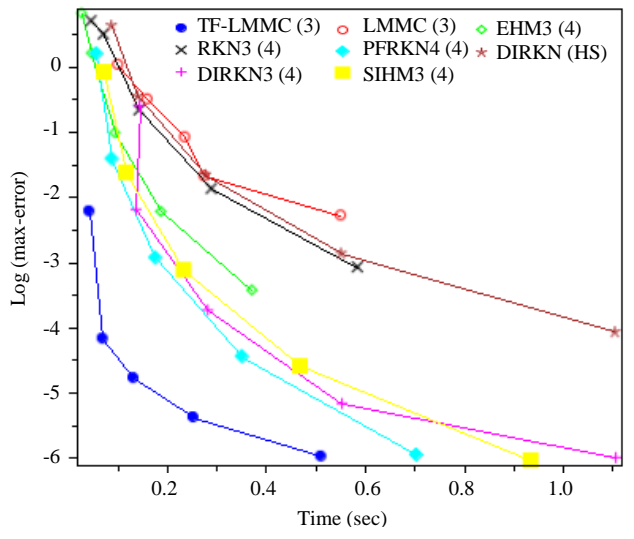

Fig. 1: The efficiency curve for TF-LMMC (3) for problem 1 with $\mathrm{T}_{\text {end }}=10^{4}$ and $\mathrm{h}=0.9 / 2^{\mathrm{i}}$ for $\mathrm{i}=1, \ldots, 5$

Problem 4; Homogeneous problem from Chakravarti and Worland (1971):

$$
y^{\prime \prime}(x)=-y(x), y(0)=0, y^{\prime \prime}(0)=1
$$

Exact solution is $y(x)=\sin (x)$. The fitted frequency is $\omega=1$.

Problem 5; Homogenous given by Attili et al. (2006):

$$
y^{\prime \prime}(x)=-64 y(x), y(0)=\frac{1}{4}, y^{\prime}(0)=-\frac{1}{2}
$$

Exact solution is:

$$
y=\frac{\sqrt{17}}{16} \sin (8 x+\theta), \theta=\pi-\tan ^{-1}(4)
$$

The fitted frequency is $\omega=8$.

Problem 6; In homogeneous equation studied by Papadopoulos et al. (2009):

$$
\begin{gathered}
y^{\prime \prime}(x)-\omega^{2} y(x)+\left(\omega^{2}-1\right) \sin (x), y(0)=1, y^{\prime}(0)=\omega+1 \\
y(x)=\cos (\omega x)+\sin (\omega x)+\sin (x)
\end{gathered}
$$

Exact solution is $y(x)=\cos (\omega x)+\sin (\omega x)+\sin (x)$. The fitted frequency is $\omega=10$. The following are the notation used in Fig. 1-12. The numerical results are shown in efficiency curves in Fig. 1-6 for TF-LMMC $(3,4)$ are shown in Fig. 7-12.

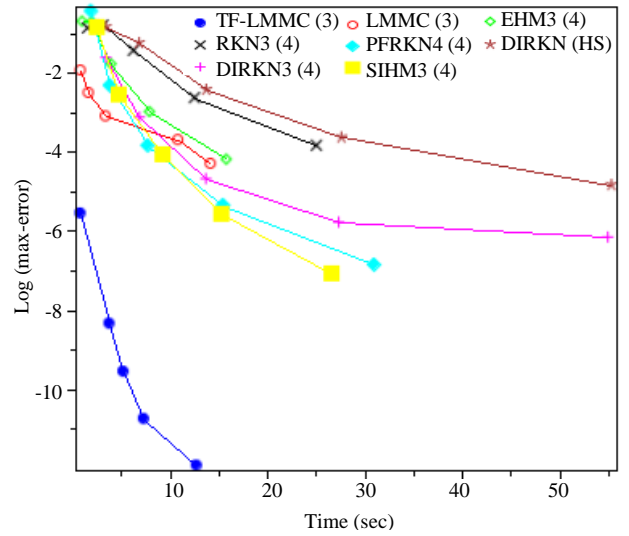

Fig. 2: The efficiency curve for TF-LMMC (3) for problem 2 with $\mathrm{T}_{\text {end }}=10^{4}$ and $\mathrm{h}=0.125 / 2^{\mathrm{i}}$ for $\mathrm{i}=2, \ldots, 6$

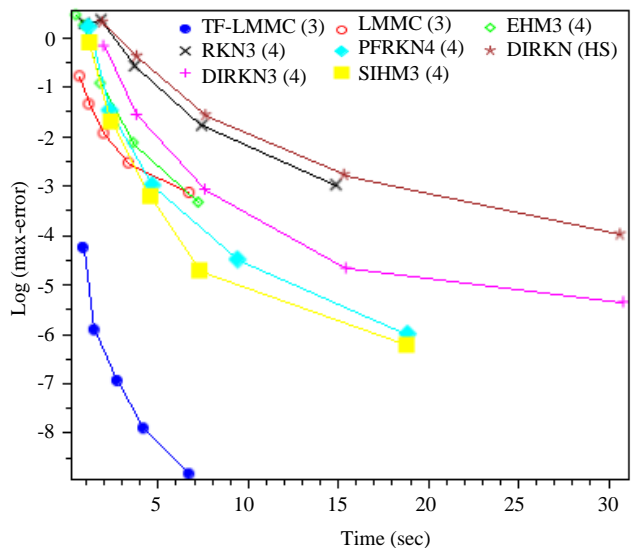

Fig. 3: The efficiency curve for TF-LMMC (3) for problem 3 with $\mathrm{T}_{\text {end }}=10^{4}$ and $\mathrm{h}=0.125 / 2^{\mathrm{i}}$ for $\mathrm{i}=1, \ldots, 5$

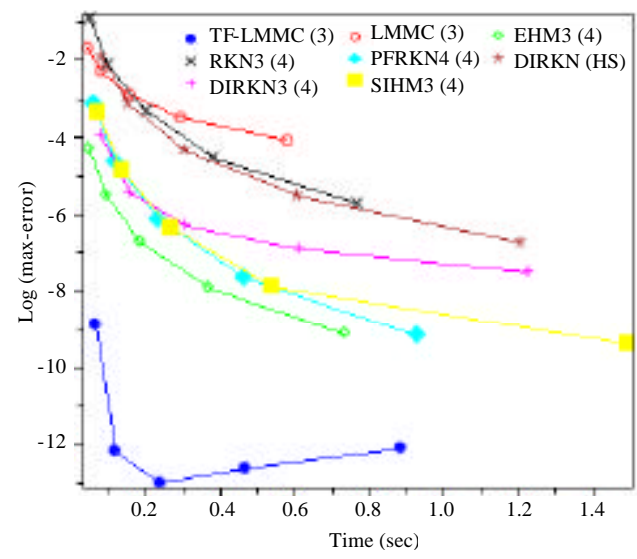

Fig. 4: The efficiency curve for TF-LMMC (3) for problem 4 with $\mathrm{T}_{\text {end }}=10^{4}$ and $\mathrm{h}=0.5 / 2^{\mathrm{i}}$ for $i=1, \ldots, 5$ 


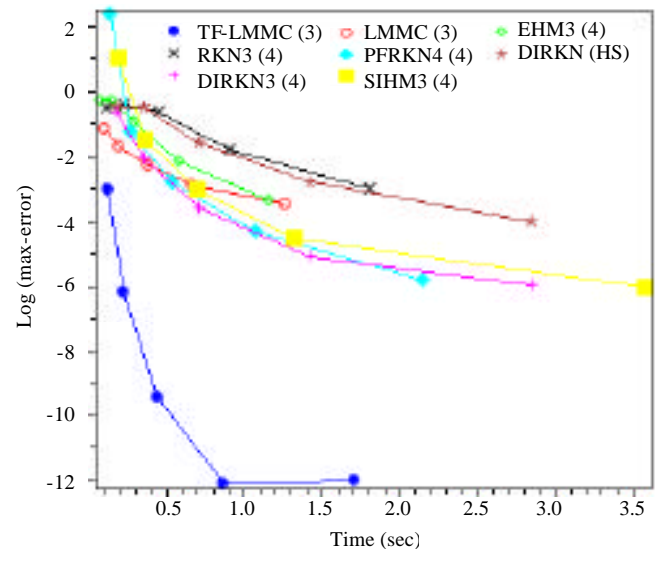

Fig. 5: The efficiency curve for TF-LMMC (3) for problem 5 with $\mathrm{T}_{\text {end }}=10^{4}$ and $\mathrm{h}=0.1 / 2^{\mathrm{i}}$ for $\mathrm{i}=3, \ldots, 7$

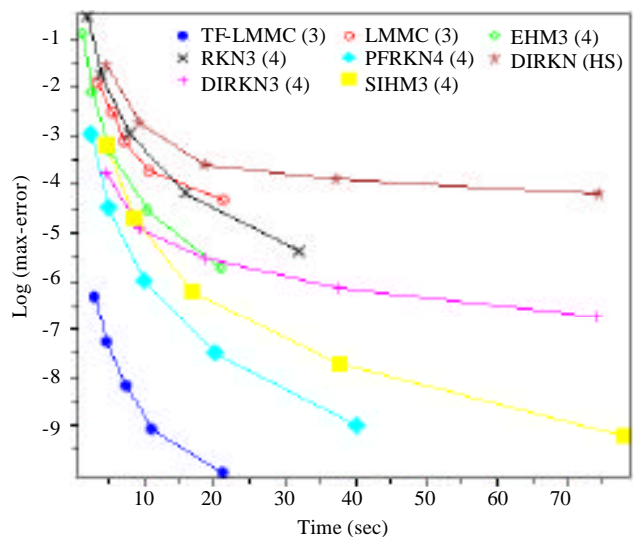

Fig. 6: The efficiency curve for TF-LMMC (3) for problem 6 with $\mathrm{T}_{\text {end }}=10^{4}$ and $\mathrm{h}=0.125 / 2^{\mathrm{i}}$ for $\mathrm{i}=3, \ldots, 7$

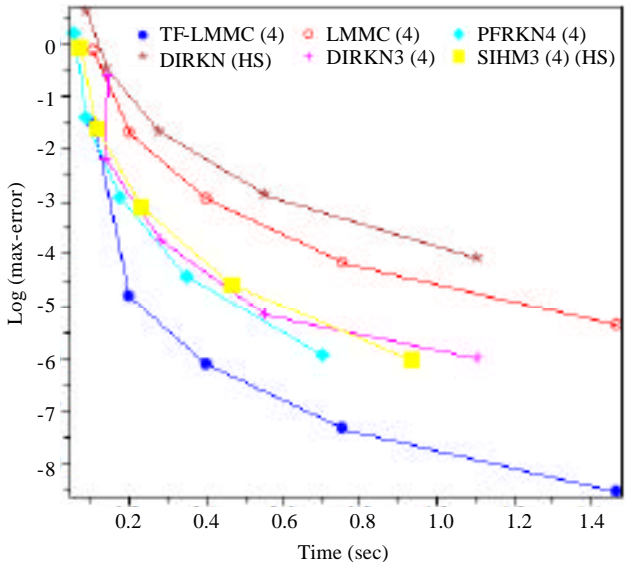

Fig. 7: The efficiency curve for TF-LMMC (4) for Problem 1 with $\mathrm{T}_{\text {end }}=10^{4}$ and $\mathrm{h}=0.9 / 2^{\mathrm{i}}$ for $\mathrm{i}=1, \ldots, 5$

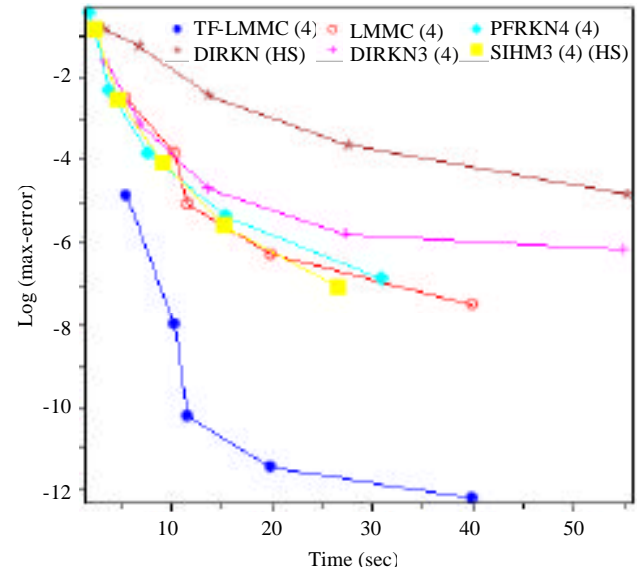

Fig. 8: The efficiency curve for TF-LMMC (4) for problem 2 with $\mathrm{T}_{\text {end }}=10^{4}$ and $\mathrm{h}=0.125 / 2^{\mathrm{i}}$ for $\mathrm{i}=2, \ldots, 6$

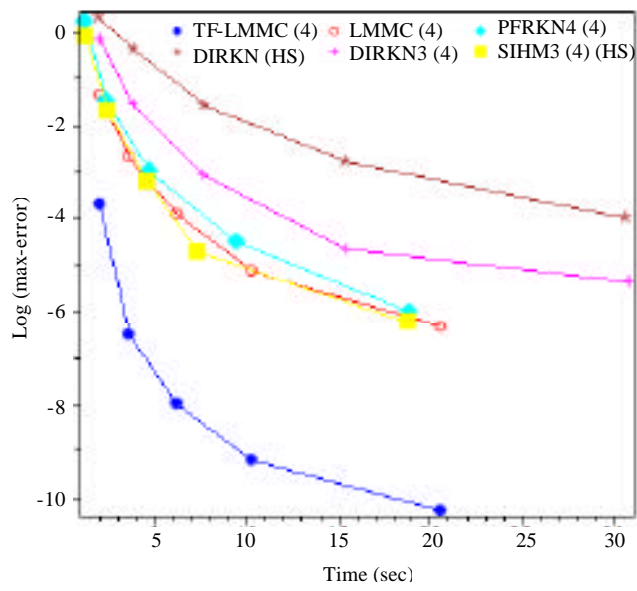

Fig. 9: The efficiency curve for TF-LMMC (4) for problem 3 with $\mathrm{T}_{\text {end }}=10^{4}$ and $\mathrm{h}=0.125 / 2^{\mathrm{i}}$ for $\mathrm{i}=1, \ldots, 5$

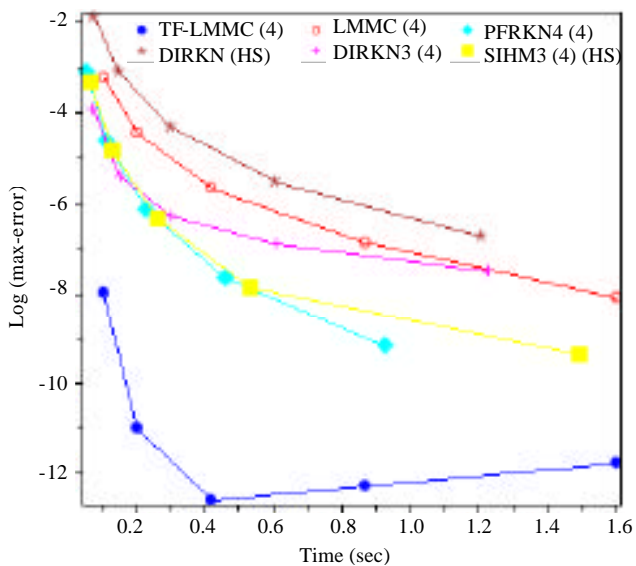

Fig. 10: The efficiency curve for TF-LMMC (4) for problem 4 with $\mathrm{T}_{\text {end }}=10^{4}$ and $\mathrm{h}=0.5 / 2^{\mathrm{i}}$ for $\mathrm{i}=1, \ldots, 5$ 


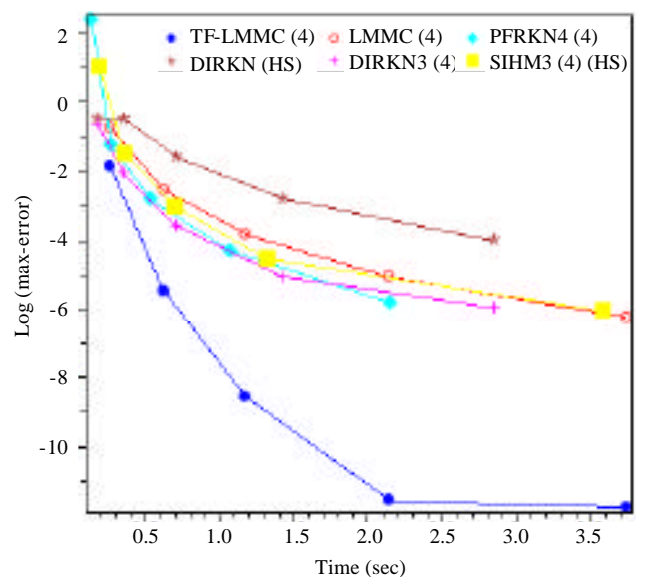

Fig. 11: The efficiency curve for TF-LMMC (4) for problem 5 with $\mathrm{T}_{\text {end }}=10^{4}$ and $\mathrm{h}=0.1 / 2^{\mathrm{i}}$ for $\mathrm{i}=3, \ldots, 7$

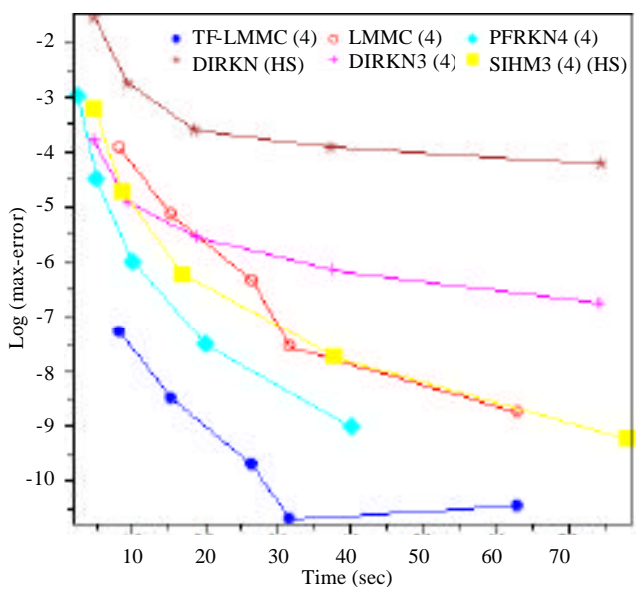

Fig. 12: The efficiency curve for TF-LMMC (4) for problem 6 with $\mathrm{T}_{\text {end }}=10^{4}$ and $\mathrm{h}=0.125 / 2^{\mathrm{i}}$ for $i=3, \ldots, 7$

\section{CONCLUSION}

In this study, we developed linear multistep methods with extra derivatives using collocation technique of order three (LMMC (3)) and four (LMMC (4)) and modified version of the methods which are Trigonometrically-Fitted Linear Multistep Method denoted as (TF-LMMC $(3,4))$, respectively.

The results show a significant improvement in accuracy for the method when adapted to trigonometrically-fitted. Numerical results for LMMC (3) which has order three is as comparable as other existing methods which are of order four and numerical results for LMMC (4) which is order four is slightly better than other existing methods in comparisons. Hence, having extra derivtives in the multistep method do improved the accuracy of the methods. However, TF-LMMC $(3,4)$ are clearly superior in solving special second order ODEs with oscillatory solutions, since, it involves lesser computational time and better accuracy. Although, TF-LMMC (4) is an implicit method, the method is more accurate in terms of accuracy and need lesser time to do the computation compared to the existing the methods in comparisons. We can conclude that TF-LMMC $(3,4)$ are very promising methods for integrating oscillating problems.

\section{ACKNOWLEDGEMENT}

The researchers would like to thank Universiti Putra Malaysia for funding this research through Putra Research Grant vote number 943500.

\section{NOMANCLATURE}

\begin{tabular}{|c|c|}
\hline & $=$ Ste \\
\hline Tethod & $=$ Method employed \\
\hline & $=$ Size of interval \\
\hline $\mathrm{MC} \mathrm{(3)}$ & $\begin{aligned}= & \text { Trigonometrically-Fitted Linear } \\
& \text { Multistep Method with Collocation } \\
& \text { method of order three developed in this } \\
& \text { chapter }\end{aligned}$ \\
\hline $\mathrm{MMC}(3)$ & $\begin{aligned} &= \text { A Linear Multistep Method with } \\
& \text { collocation method of order three } \\
& \text { developed in this chapter }\end{aligned}$ \\
\hline F-LM & $\begin{aligned}= & \text { Trigonometrically-Fitted Linear } \\
& \text { Multistep Method with Collocation } \\
& \text { method of order four developed in this } \\
& \text { chapter }\end{aligned}$ \\
\hline LMMC (4) & $\begin{aligned}= & \text { A Linear Multistep Method with } \\
& \text { Collocation method of order four } \\
& \text { developed in this chapter }\end{aligned}$ \\
\hline EHM3 (4) & $\begin{aligned}= & \text { Explicit three-stage fourth-order Hybrid } \\
& \text { Method derived by Franco }(2006)\end{aligned}$ \\
\hline RKN3 (4) & $\begin{aligned}= & \text { Explicit three-stage fourth-order RKN } \\
& \text { method by Hairer et al. }(2010)\end{aligned}$ \\
\hline PFRKN4 (4) & $\begin{aligned}= & \text { Explicit four-stage fourth-order } \\
& \text { Phase-fitted RKN method by } \\
& \text { Papadopoulos et al. }(2009)\end{aligned}$ \\
\hline DIRKI & $\begin{aligned} &= \text { Diagonally implicit three-stage } \\
& \text { fourth-order RKN method derived by } \\
& \text { Sommeijer (1987) }\end{aligned}$ \\
\hline DIRKN3 (4) & $\begin{aligned}= & \text { Diagonally implicit three-stage } \\
& \text { fourth-order RKN method derived by } \\
& \text { Senu et al. }(2010)\end{aligned}$ \\
\hline SIHN & $\begin{aligned}= & \text { Semi-implicit three-stage fourth-order } \\
& \text { hybrid method developed by } \\
& \text { Ahmad et al. }(2013 \mathrm{a}, \mathrm{b})\end{aligned}$ \\
\hline
\end{tabular}




\section{REFERENCES}

Ahmad, S.Z., F. Ismail, N. Senu and M. Suleiman, 2013a. Semi implicit hybrid methods with higher order dispersion for solving oscillatory problems. Abstract Appl. Anal., 2013: 1-10.

Ahmad, S.Z., F. Ismail, N. Senu and M. Suleiman, 2013b. Zero-dissipative phase-fitted hybrid methods for solving oscillatory second order ordinary differential equations. Appl. Math. Comput., 219: 10096-10104.

Attili, B.S., K. Furati and M.I. Syam, 2006. An efficient implicit Runge-Kutta method for second order systems. Appl. Math. Comput., 178: 229-238.

Chakravarti, P.C. and P.B. Worland, 1971. A class of self-starting methods for the numerical solution ofy? $=\mathrm{f}(\mathrm{x}, \mathrm{y})$. BIT. Numer. Math., 11: 368-383.

Coleman, J.P., 2003. Order conditions for a class of two-step methods for $y ?=\mathrm{f}(\mathrm{x}, \mathrm{y})$. IMA. J. Numer. Anal., 23: 197-220.

Dormand, J.R., M.E.A. El-Mikkawy and P.J. Prince, 1987. Families of Runge-Kutta-Nystrom formulae. IMA. J. Numer. Anal., 7: 235-250.

Franco, J.M., 1995. An explicit hybrid method of Numerov type for second-order periodic initial-value problems. J. Comput. Appl. Math., 59: 79-90.

Franco, J.M., 2006. A class of explicit two-step hybrid methods for second-order IVPs. J. Comput. Appl. Math., 187: 41-57.

Guo, Y.J.L., 2007. A third order equation arising in the falling film. Taiwanese J. Math., 11: 637-643.

Hairer, E., S.P. Norsett and G. Wanner, 2010. Solving Ordinary Differential Equations I: Nonstiff Problems. 2nd Edn., Springer Verlag GmbH, Heidelberg, Germany, ISBN:9783642081583, Pages: 528.

Henrici, P., 1962. Discrete Variable Methods in Ordinary Differential Equation. 1st Edn., Wiley and Sons, New York.

Jator, S.N., 2008. Numerical integrators for fourth order initial and boundary value problems. Intl. J. Pure Appl. Math., 47: 563-576.
Kosti, A.A., Z.A. Anastassi and T.E. Simos, 2012. An optimized explicit Runge-Kutta-Nystrom method for the numerical solution of orbital and related periodical initial value problems. Comput. Phys. Commun., 183: 470-479.

Lambert, J.D. and I.A. Watson, 1976. Symmetric multistep methods for periodic initial value problems. IMA J. Applied Math., 18: 189-202.

Lambert, J.D., 1973. Computational Methods in Ordinary Differential Equations. Wiley Publishing Company, Hoboken, New Jersey, USA., ISBN:9780471511946, Pages: 278 .

Papadopoulos, D.F., Z.A. Anastassi and T.E. Simos, 2009. A phase-fitted Runge-Kutta-Nystrom method for the numerical solution of initial value problems with oscillating solutions. Comput. Phys. Commun., 180: 1839-1846.

Samat, F., F. Ismail and M.B. Suleiman, 2011. A variable step-size exponentially fitted explicit hybrid method for solving oscillatory problems. Intl. J. Math. Math. Sci., 2011: 1-17.

Senu, N., M. Suleiman, F. Ismail and M. Othman, 2010. A fourth-order diagonally implicit Runge-Kutta-Nystrom method with dispersion of high order. Proceedings of the 4th International Conference on Applied Mathematics, Simulation, Modelling (ASM'10), July 22-25, 2010, World Scientific and Engineering Academy and Society (WSEAS), Stevens Point, Wisconsin, USA., ISBN: 978-960-474-210-3, pp: 78-82.

Sommeijer, B.P., 1987. A note on a diagonally implicit Runge-Kutta-Nystrom method. J. Comput. Appl. Math., 19: 395-399.

Stiefel, E. and D.G. Bettis, 1969. Stabilization of Cowells method. Numerische Math., 13: 154-175.

Yap, L.K., F. Ismail and N. Senu, 2014. An accurate block hybrid collocation method for third order ordinary differential equations. J. Appl. Math., 2014: 1-9. 